\title{
Scalarizations for Maximization with Respect to Polyhedral Cones
}

\author{
H.W Corley \\ Center On Stochastic Modeling, Optimization, \& Statistics (COSMOS), The University of Texas at Arlington, \\ Arlington, Texas, United States \\ Email: corley@uta.edu
}

\begin{abstract}
Efficient points are obtained for cone-ordered maximizations in $R^{n}$ using the method of scalarization. Various scalarizations are presented for ordering cones in general and then for the important special case of polyhedral cones. For polyhedral cones, it is shown how to find vectors in the positive dual cone that are needed for a scalarized objective function. Instructive examples are presented.
\end{abstract}

Keywords: Multiobjective optimization, polyhedral cones, cone maximization, Pareto maximization, scalarization.

\section{Introduction}

Multiobjective optimization is applied in various fields of science, engineering, and economics when decisions involve two or more conflicting objectives. For example, a pharmaceutical company may wish to determine a dose for a new drug that would maximize its therapeutic benefits while minimizing its deleterious side effects. The result would be a multiobjective optimization problem. In many other situations, however, a decision may not be adequately characterized by such standard criteria as Pareto optimality, goal programming, or lexicographic maximization. For this reason, cone-ordered maximization was developed, of which the previously mentioned criteria are special cases. Early results for cone-ordered maximizations, formulated with various degrees of abstraction, include optimality conditions by Hurwicz [1], Neustadt [2], Ritter [3], Craven [4], Yu [5] Borwein [6], Christotspeit [7], and Corley [8], as well as a duality theory by Corley [9]. Such results are difficult to implement, however. For this reason, scalarization is the method of choice for actually obtaining a numerical solution to a cone-ordered maximization.

A scalarization is an optimization problem with a single scalar objective function for which an optimal solution solves the cone-ordered problem. Examples are the scalarizations of Borwein [6] and Jahn [10] in terms of the dual cone and that of Soland [11] for Pareto maximization involving both the dual cone and aspiration levels for the individual objective functions. References [12-18] consolidate the theory and solution techniques for cone-ordered maximizations. However, specific results for polyhedral cones are scarce. For such cones, Yu [5] proposes a specialized scalarization based on finding cone extreme points. Sawaragi et al. [1985] reduce the problem to finding Pareto efficient points of a set in the objective function's image space. More recently, for example, Gutierrez, et al. [19] define a scalarization by perturbing the same matrix.

We consider a decision problem with an n-dimensional objective function, where the optimization criterion is maximization with respect to a partial order induced by a pointed convex cone in $R^{n}$. The purpose here is to present scalarizations for such problems in general, with the emphasis on polyhedral cones. Methods are also developed for finding dual vectors of a polyhedral cone as required for scalarized objective functions. One such approach involves linear programming. The paper is organized as follows. In Section 2 the requisite preliminaries concerning cones are established, while the notion of coneordered maximization is defined in Section 3. In Section 4 various scalarizations are presented. Section 5 then addresses the problem of obtaining the required dual vectors for polyhedral cones. Finally, in Section 6, instructive examples involving polyhedral cones are solved by scalarization. Conclusions are offered in Section 7. 


\section{Cone Preliminaries}

Notationally, any vector $\mathbf{x}=\left(x_{1}, \ldots, x_{m}\right) \in R^{m}$ or $\mathbf{y}=\left(y_{1}, \ldots, y_{n}\right) \in R^{n}$ will be considered a column vector, and $\mathbf{0}$ will be a column vector of zeros of appropriate dimension. A nonempty set $C \subset R^{n}$ is a cone if $\lambda \mathbf{y} \in C$ for all $\mathbf{y} \in C$ and all $\lambda>0$. A cone $C$ is said to be nontrivial if $C \neq\{\mathbf{0}\}$, pointed if $C \cap-C=\{\mathbf{0}\}$, and convex if $\lambda_{1} \mathbf{y}_{1}+\lambda_{2} \mathbf{y}_{2} \in C$ for all $\mathbf{y}_{1}, \mathbf{y}_{2} \in C$ and all $\lambda_{1}, \lambda_{2}>0 . \quad C$ is a nontrivial, closed, convex, polyhedral cone if and only if there exists a finite nonempty set of nonzero vectors $G=\left\{\mathbf{b}_{1}, \ldots, \mathbf{b}_{r}\right\} \subset R^{n}$ that conically span $C$; i.e., $C=\left\{\sum_{j=1}^{r} \alpha_{j} \mathbf{b}_{j}: \alpha_{j} \geq 0, j=1, \ldots, r\right\}$. In this paper, any cone $C$ will be assumed nontrivial, pointed, and convex, so any polyhedral cone $C$ here will be pointed unless otherwise stated. Pointedness is necessary in the next section for defining a partial order in terms of $C$.

In general a cone does not contain $\{\mathbf{0}\}$, but a polyhedral cone generated by $G=\left\{\mathbf{b}_{1}, \ldots, \mathbf{b}_{r}\right\}$ does. $G$ is called the set of generators for $C$, which is simply the conical hull of $G$. If no subset of $G$ conically spans $C$, the elements of $G$ are said to be conically independent. In other words, no $\mathbf{b}_{j}$ can be expressed as a conical combination of the other members of $G$. If $\mathbf{b}_{1}, \ldots, \mathbf{b}_{r}$ are conically independent, they are called basic generators, or extreme directions, for the polyhedral cone $C$. They represent a minimal generating set for the cone and are unique within a positive scaling. In $R^{n}$ the number $r$ of basic generators for a polyhedral cone may be less than, equal to, or greater than $n$.

Supplementing the results in [20] and [21], a consequence of the polyhedral cones here being pointed is next established.

Definition 1. The nonzero vectors $\mathbf{b}_{1}, \ldots, \mathbf{b}_{r}$ in $R^{n}$ are said to be conically homogeneous if and only if the equation $\sum_{j=1}^{r} \alpha_{j} \mathbf{b}_{j}=\mathbf{0}$ for scalars $\alpha_{j} \geq 0, j=1, \ldots, r$ implies that $\alpha_{j}=0, j=1, \ldots, r$.

Theorem 2. Let $\mathbf{b}_{1}, \ldots, \mathbf{b}_{r}$ be generators for a polyhedral cone $C$ in $R^{n}$, which is not necessarily pointed. Then $C$ is pointed if and only if $\mathbf{b}_{1}, \ldots, \mathbf{b}_{r}$ are conically homogeneous.

Proof. Consider the generators $\mathbf{b}_{1}, \ldots, \mathbf{b}_{r}$ for $C$. To prove that pointedness implies conical homogeneity, suppose that $\mathbf{b}_{1}, \ldots, \mathbf{b}_{r}$ are not conically homogeneous. Then there exist scalars $\alpha_{j} \geq 0, j=1, \ldots, r$, with $\alpha_{k}>0$ for some $k$ and $\sum_{j=1}^{r} \alpha_{j} \mathbf{b}_{j}=\mathbf{0}$. Write

$$
\sum_{\substack{j=1 \\ j \neq k}}^{r} \alpha_{j} \mathbf{b}_{j}=-\alpha_{k} \mathbf{b}_{k}
$$

Thus the nonzero right side of (1) is in $-C$, while the left side in $C$. Hence $C$ cannot be pointed.

Then there exists a nonzero $\sum_{j=1}^{r} \alpha_{j} \mathbf{b}_{j}$ in $C$ with $\alpha_{j} \geq 0, j=1, \ldots, r$, and some $\alpha_{k}>0$ such that $\sum_{j=1}^{r} \alpha_{j} \mathbf{b}_{j}$ is in $-C$. Since $-\mathbf{b}_{1}, \ldots,-\mathbf{b}_{r}$ are obviously generators for $-C$, it follows that there exists a nonzero $\sum_{j=1}^{r} \beta_{j}\left(-\mathbf{b}_{j}\right)$ in $-C$ with $\beta_{j} \geq 0, j=1, \ldots, r$, and some $\beta_{l}>0$ such that $\sum_{j=1}^{r} \alpha_{j} \mathbf{b}_{j}=\sum_{j=1}^{r} \beta_{j}\left(-\mathbf{b}_{j}\right)$. Hence $\sum_{j=1}^{r}\left(\alpha_{j}+\beta_{j}\right) \mathbf{b}_{j}=\mathbf{0}$, with $\left(\alpha_{j}+\beta_{j}\right) \geq 0$ but not all zero. Thus $\mathbf{b}_{1}, \ldots, \mathbf{b}_{r}$ are not conically homogeneous, and the result follows.

Conical independence is not needed in Theorem 2. However, basic generators are conically independent by definition. From Theorem 2, the assumption that any polyhedral cone $C$ here be pointed is equivalent to its generators $\mathbf{b}_{1}, \ldots, \mathbf{b}_{r}$ being assumed conically homogenous. Any generators $\mathbf{b}_{1}, \ldots, \mathbf{b}_{r}$ used in this paper will be assumed basic, though the results apply to any conically 
homogenous generators. The following immediate corollary of Theorem 2 illustrates a similarity that basic generators for a pointed polyhedral cone share with basis vectors for $R^{n}$, which are linearly independent and hence linearly homogenous for $\alpha_{j}$ not necessarily all nonnegative.

Corollary 3. Let $\mathbf{b}_{1}, \ldots, \mathbf{b}_{r}$ be basic generators for a pointed polyhedral cone $C$ in $R^{n}$. Then $\mathbf{b}_{1}, \ldots, \mathbf{b}_{r}$ are both conically independent and conically homogeneous.

In addition to the generator set approach for representing a polyhedral cone $C$, there is also a generator matrix approach. As noted in [13], for any polyhedral cone $C$ in $R^{n}$ there exists an $t \times n$ matrix A with kernel $\left\{\mathbf{y} \in R^{n}: \mathbf{A y}=\mathbf{0}\right\}=\{\mathbf{0}\}$ for which $C=\left\{\mathbf{y} \in R^{n}: \mathbf{A y} \geq \mathbf{0}\right\}$. The condition that the kernel of $\mathbf{A}$ be $\{\mathbf{0}\}$ has a similar role to the condition that the generators be conically homogeneous. Indeed, it is easily shown that $C$ is pointed if and only of the kernel of $\mathbf{A}$ is $\{\mathbf{0}\}$. The generator-set representation and the generator-matrix representation for polyhedral cones are formally shown to be equivalent in the Farkas-Minkowski-Weyl theorem of [22]. In the former representation, a polyhedral cone $C$ is the conical hull of its generators $\mathbf{b}_{1}, \ldots, \mathbf{b}_{r}$. In the latter, $C$ is the intersection of $t$ half-spaces defined by $\mathbf{A y} \geq \mathbf{0}$. However, the generator set approach may be the more intuitive one for modeling a polyhedral cone $C$ in a higher dimensional multiple objective problem. Generator sets appear more amenable to a trial and error process for specifying an appropriate ordering cone than do generator matrices.

The notion of a dual cone is next defined.

Definition 4. Let $C$ be a cone in $R^{n}$. The dual cone of $C$ is defined to be the set $\left\{\mathbf{d}=\left(d_{1}, \ldots, d_{n}\right) \in R^{n}: \mathbf{d}^{\mathrm{T}} \mathbf{y}=\sum_{j=1}^{n} d_{j} y_{j} \geq 0, \forall \mathbf{y}=\left(y_{1}, \ldots, y_{n}\right) \in C \backslash\{\mathbf{0}\}\right\}$.

A vector $\mathbf{d}$ in the dual cone of $C$ that further satisfies $\mathbf{d}^{\mathrm{T}} \mathbf{y}>0$ for all $\mathbf{y} \in C \backslash\{\mathbf{0}\}$ is said to be a positive dual vector on $C$. Write $C^{+}=\left\{\mathbf{d} \in R^{n}: \mathbf{d}^{\mathrm{T}} \mathbf{y}>0, \forall \mathbf{y}=\left(y_{1}, \ldots, y_{n}\right) \in C \backslash\{\mathbf{0}\}\right\}$. The following two results are stated for future reference. Result 5 is proved in [6] for more general spaces than $R^{n}$, while Result 6 is immediate.

Result 5. For any closed cone $C$ in $R^{n}$ there exists $\mathbf{d} \in C^{+}$.

Result 6. Let $C$ be a polyhedral cone in $R^{n}$ with basic generators $\mathbf{b}_{1}, \ldots, \mathbf{b}_{r}$, and let $\mathbf{d} \in R^{n}$. Then $\mathbf{d} \in C^{+}$if and only if $\mathbf{d}^{\mathrm{T}} \mathbf{b}_{i}>0, i=1, \ldots, r$.

\section{Cone Maximization}

Any pointed convex cone $C$ in $R^{n}$ determines a partial order $\leq_{C}$ on $R^{n}$ as follows. For any $\mathbf{y}_{1}, \mathbf{y}_{2} \in R^{n}$, we say that $\mathbf{y}_{1} \leq_{C} \mathbf{y}_{2} \quad\left(\right.$ or $\left.\mathbf{y}_{2} \geq_{C} \mathbf{y}_{1}\right)$ if $\mathbf{y}_{2}-\mathbf{y}_{1} \in C$. Write $\mathbf{y}_{1}<_{C} \mathbf{y}_{2}\left(\right.$ or $\left.\mathbf{y}_{2}>_{C} \mathbf{y}_{1}\right)$ if $\mathbf{y}_{1} \leq_{C} \mathbf{y}_{2}$ and $\mathbf{y}_{1} \neq \mathbf{y}_{2}$, i.e., if $\mathbf{y}_{2}-\mathbf{y}_{1} \in C \backslash\{\mathbf{0}\}$. In particular, we say that $\mathbf{y}_{2}$ dominates $\mathbf{y}_{1}$ if $\mathbf{y}_{1}<_{C} \mathbf{y}_{2}$. A vector $\mathbf{y}^{*} \in Y \subset R^{n}$ is said to be nondominated on $Y$ if there is no $\mathbf{y} \in Y$ such that $\mathbf{y}^{*}<_{C} \mathbf{y}$, or equivalently, that $Y \cap\left(C+\mathbf{y}^{*}\right)=\{\mathbf{0}\}$. Define the set $\max _{C} Y$ as the set containing all nondominated vectors in $Y$ with respect to the cone $C$. Consider now the objective functions $f_{i}: X \subset R^{m} \rightarrow R^{1}, i=1, \ldots, n$, and the column vector $f(\mathbf{x})=\left(f_{1}(\mathbf{x}), \ldots, f_{n}(\mathbf{x})\right)$. For the cone $C \subset R^{n}$, write the cone maximization with respect to $C$ of $f(\mathbf{x})$ on the feasible set $X$ as

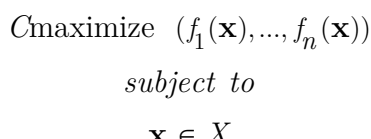

Problem (2) involves finding $\mathbf{x}^{*} \in X \subset R^{m}$, such that $f\left(\mathbf{x}^{*}\right) \in \max _{C} f(X)$ for $f(X)=\bigcup_{\mathbf{x} \in X}\{f(\mathbf{x})\}$. Such an $\mathbf{x}^{*}$ is called a C-efficient point (or just efficient point) for problem (2), and the set of $\left(f_{1}\left(\mathbf{x}^{*}\right), \ldots, f_{n}\left(\mathbf{x}^{*}\right)\right)$ for all efficient points $\mathbf{x} *$ is called the C-efficient frontier (or just efficient frontier) of problem (2). The set of efficient points for (2) is sometimes written $E[f(X), C]$. When $X$ is convex, the 
function $f(\mathbf{x})$ is said to be C-concave on $X$ if and only if $f\left[\lambda \mathbf{x}_{1}+(1-\lambda) \mathbf{x}_{2}\right] \geq_{C} \lambda f\left(\mathbf{x}_{1}\right)+(1-\lambda) f\left(\mathbf{x}_{2}\right)$ for all $\mathbf{x}_{1}, \mathbf{x}_{2} \in X$ and $\lambda \in[0,1]$.

A more restrictive type of efficient point is defined in [6] using tangent cones. Let $Y \subset R^{n}$ and $\mathbf{y}_{0} \in \bar{Y}$, the closure of $Y$. The tangent cone $T\left(Y, \mathbf{y}_{0}\right)$ to $Y$ at $Y$ is the set of limits of the form $\mathbf{y}=\lim _{n \rightarrow \infty} \lambda_{n}\left(\mathbf{y}_{n}-\mathbf{y}_{0}\right)$, where $\lambda_{1}, \lambda_{2}, \ldots$ is sequence of non-negative real numbers and $\mathbf{y}_{1}, \mathbf{y}_{2}, \ldots$ is a sequence in $Y$ with limit $\mathbf{y}_{0}$. Then a C-efficient point $\mathbf{x}^{*} \in X$ is a proper C-efficient point (or just proper efficient point) for problem (2) if $C \cap T\left[f(A)-C, f\left(\mathbf{x}^{*}\right)\right]=\{\mathbf{0}\}$ and an improper efficient point otherwise. Geometrically, an improper efficient point $\mathbf{x}^{*}$ is an efficient point for which $f\left(\mathbf{x}^{*}\right)$ can be approached in $f(A)-C$ from directions within $C+f\left(\mathbf{x}^{*}\right)$. Operationally, improper efficient points are those efficient points that cannot be obtained via scalarization for some $\mathbf{d} \in C^{+}$in problem (5) of the next section.

To illustrate proper and improper efficient points for problem (2) with $m=n=2$, let $f: R^{2} \rightarrow R^{2}$ be the identity function $f\left(x_{1}, x_{2}\right)=\left(x_{1}, x_{2}\right)$, let $X=\left\{\left(x_{1}, x_{2}\right): x_{1}^{2}+x_{2}^{2} \leq 1, x_{1} \geq 0, x_{2} \geq 0\right\}$, and let $C=\left\{\left(x_{1}, x_{2}\right): x_{2} \geq 0, x_{1} \geq x_{2}\right\}$. Applying the definition of $\max _{C} f(X)$ in $R^{2}$ and using the fact that $\mathbf{x}^{*} \in E[f(X), C]$ is equivalent to the condition $f(X) \cap\left[C+f\left(\mathbf{x}^{*}\right)\right]=\{\mathbf{0}\}$, it readily follows that $E[f(X), C]=\left\{\left(x_{1}, x_{2}\right): x_{1}^{2}+x_{2}^{2}=1, x_{1} \geq 0, x_{2} \geq 0\right\}$. The only improper efficient point is $(0,1)$.

As a special case of problem (2), let $C$ be the polyhedral cone $P=\left\{\left(y_{1}, \ldots, y_{n}\right): y_{i} \geq 0, i=1, \ldots, n\right\}$, which is termed the Pareto cone. A set of basic generators is the standard orthonormal basis $\mathbf{e}_{1}=(1,0, \ldots, 0), \ldots, \mathbf{e}_{n}=(0,0, \ldots, 1)$ for $R^{2}$, so $\mathbf{d} \in P^{+}$if and only if $d_{i}>0, i=1, \ldots, n$. For the cone $P$, problem (2) becomes the Pareto maximization problem

$$
\begin{gathered}
\text { Pmaximize }\left(f_{1}(\mathbf{x}), \ldots, f_{n}(\mathbf{x})\right) \\
\text { subject to } \\
\mathbf{x} \in X
\end{gathered}
$$

A feasible point $\mathbf{x}^{*} \in X$ is a Pareto maximum, or Pareto efficient point, of problem (3) if and only if there is no $\mathbf{x} \in X$ such that $\left(f_{1}\left(\mathbf{x}^{*}\right), \ldots, f_{n}\left(\mathbf{x}^{*}\right)\right){ }_{P}\left(f_{1}(\mathbf{x}), \ldots, f_{n}(\mathbf{x})\right)$. In other words, $\mathbf{x}^{*} \in X$ is Pareto efficient if and only if there is no $\mathbf{x} \in X$ for which $f_{i}\left(\mathbf{x}^{*}\right) \leq f_{i}(\mathbf{x}), i=1, \ldots, n$, and $f_{k}\left(\mathbf{x}^{*}\right)<f_{k}(\mathbf{x})$ for some $k$. In the Pareto case, an improper efficient point is an efficient point for which the marginal gain for some objective function $f_{i}$, relative to the marginal loss in all the other objective functions, is unbounded from above [23].

Consider now problem (2), where $C$ is a polyhedral cone. Then (2) can be transformed into a Pareto maximization problem in $R^{t}$ if the $t \times n$ matrix $\mathbf{A}$ with kernel $\{0\}$ is known for which $C=\left\{\mathbf{y} \in R^{n}: \mathbf{A y} \geq \mathbf{0}\right\}$. In this case, problem (2) can be written as

$$
\begin{gathered}
\text { Pmaximize } \mathbf{A} f(\mathbf{x}) \\
\text { subject to } \\
\mathbf{x} \in X
\end{gathered}
$$

from a result in [13]. To see (4), let $\mathbf{x}^{*} \in X$. Then $\mathbf{x}^{*}$ does not solve (2) if and only if there exists $\hat{\mathbf{x}} \in X$ such that $f(\hat{\mathbf{x}})>_{C} f\left(\mathbf{x}^{*}\right)$. The kernel of $\mathbf{A}$ is $\{\mathbf{0}\}$, so $f(\hat{\mathbf{x}})-f\left(\mathbf{x}^{*}\right) \in C \backslash\{\mathbf{0}\} \subset R^{n}$ if and only if $\mathbf{A}\left[f(\hat{\mathbf{x}})-f\left(\mathbf{x}^{*}\right)\right] \in P \backslash\{\mathbf{0}\} \subset R^{t}$, i.e., $\mathbf{A} f(\hat{\mathbf{x}})>_{P} \mathbf{A} f\left(\mathbf{x}^{*}\right)$. Thus $\mathbf{x}^{*}$ does not solve (4) if and only if $\mathbf{x}^{*}$ does not solve (2). It follows that when a generator matrix $\mathbf{A}$ is given, then any method for solving (3) can be used to obtain a solution for (4) and hence (2).

\section{Scalarizations}

A scalarization of problem (2) is an optimization problem with a real-valued objective function, the solutions of which also solve (2). A scalarization may involve auxiliary parameters, variables, functions, and constraints to achieve this goal. Ideally, a scalarization would be equivalent to problem (2) in the sense that the scalarization would yield all solutions and only solutions for (2). Thus it is desirable that 
every solution to (2) can be obtained with some combination of these auxiliary entities. This goal may only be possible if they are varied in a scalarization to obtain different solutions to (2). However, for some scalarizations below, obtaining all efficient points to (2) is not possible.

Scalarization for cone-ordered optimization, including the Pareto case, has been studied extensively. For example, see [23-39], which present scalarizations of varying degrees of abstraction. Few involve polyhedral cones. In this paper we present generalizations of the two fundamental scalarizations for cone-ordered maximization as given in Result 7 and Corollary 11, then specialize them to polyhedral cones. In addition, Theorem 14 is apparently new.

Result 7. Let $C$ be a closed cone in $R^{n}$, and let $\mathbf{d} \in C^{+}$. If $\mathbf{x}^{*}$ solves the scalar maximization problem

$$
\begin{gathered}
\operatorname{maximize} \mathbf{d}^{\mathrm{T}} f(\mathbf{x}) \\
\text { subject to } \\
\mathbf{x} \in X
\end{gathered}
$$

then $\mathbf{x}^{*}$ is a proper efficient point for problem (2). In addition, assume that $X$ is a convex set and $f$ is C-concave on $X$. Then $\mathbf{x}^{*}$ is a proper efficient point for problem (2) if and only if $\mathbf{x}^{*}$ solves problem (5) for some $\mathbf{d} \in C^{+}$.

Result 7 is established in [6]. The condition that $C$ be closed in Result 7, as well as in Theorem 10 and Corollary 11, is significant since (5) is proved using convex separation theorems for closed cones that require that $\mathbf{d} \in C^{+}$. A counterexample is the lexicographic cone $L$ in $R^{n}$ [13], which is nontrivial, pointed, and convex. However, $L$ is not closed and $L^{+}=\varnothing$. Result 7 generalizes the weighted sum method for Pareto optimization [20]. We apply it to polyhedral cones in the following two corollaries. However, note that the generators $\mathbf{b}_{i}=\mathbf{e}_{i}, i=1, . ., n$, for the Pareto cone in $R^{n}$ do not satisfy the condition $\mathbf{b}_{i}{ }^{\mathrm{T}} \mathbf{b}_{j}>0, i, j=1, \ldots, r$, of Corollary 8 below, which follows from Results 6 and 7 .

Corollary 8. Let $C$ be a polyhedral cone in Result 7. Then the conclusions of Result 7 hold if $C$ has basic generators $\mathbf{b}_{1}, \ldots, \mathbf{b}_{r}$ and if the vector $\mathbf{d} \in R^{n}$ satisfies $\mathbf{d}^{\mathrm{T}} \mathbf{b}_{i}>0, i=1, \ldots, r$. In particular, if $\mathbf{b}_{k}{ }^{\mathrm{T}} \mathbf{b}_{j}>0, j=1, \ldots, r$, for some $\mathbf{b}_{k}$, i.e., if the angle in $R^{n}$ between $\mathbf{b}_{k}$ and the other generators is less than $\pi / 2$, then $\mathbf{b}_{k} \in C^{+}$. Similarly, if $\mathbf{b}_{i}{ }^{\mathrm{T}} \mathbf{b}_{j}>0, i, j=1, \ldots, r$, then $\mathbf{b}_{i} \in C^{+}, i=1, \ldots, r$.

Result 7 can also be applied to a polyhedral cone specified by its generator matrix A. Since $P^{+}=\left\{\left(d_{1}, \ldots, d_{l}\right): d_{i}>0, i=1, \ldots, t\right\} \subset R^{t}$, the next result follows immediately by solving the Pareto problem (4) by Result (7).

Corollary 9. Let the $t \times n$ generator matrix $\mathbf{A}$ for the polyhedral cone $C$ have row vectors $\mathbf{a}_{1}, \ldots, \mathbf{a}_{t}$, thereby specifying the objective function of $(4)$ as $\left(\mathbf{a}_{1} f(\mathbf{x}), \ldots, \mathbf{a}_{t} f(\mathbf{x})\right) \in R^{t}$. For any $\mathbf{d}=\left(d_{1}, \ldots, d_{t}\right) \in R^{t}$ with $d_{i}>0, i=1, \ldots, t$, if $\mathbf{x} *$ solves the scalar maximization problem

$$
\begin{gathered}
\operatorname{maximize} \sum_{i=1}^{t} d_{i} \mathbf{a}_{i} f(\mathbf{x}) \\
\text { subject to } \\
\mathbf{x} \in X
\end{gathered}
$$

then $\mathbf{x}^{*}$ is a proper efficient point for problem $(2)$.

Next, Theorem 10 completely characterizes cone maximization in terms of n-dimensional parameter vectors $\mathbf{d} \in C^{+}$and $\varepsilon \in R^{n}$. It generalizes results in [11] and [15].

Theorem 10. Let $C$ be a closed cone in $R^{n}$ and let any $\mathbf{d} \in C^{+}$be given. Then $\mathbf{x}^{*}$ is an efficient point for problem (2) if and only if there exists $\varepsilon \in R^{n}$ for which $\mathbf{x}^{*}$ solves the scalar maximization problem

$$
\begin{gathered}
\text { maximize } \mathbf{d}^{\mathrm{T}} f(\mathbf{x}) \\
\text { subject to } \\
f(\mathbf{x}) \geq_{C} \varepsilon \\
\mathbf{x} \in X
\end{gathered}
$$


Proof. To prove the necessity, suppose that $\mathbf{x}^{*}$ solves problem (2). Set $\varepsilon=f\left(\mathbf{x}^{*}\right)$ in problem (7) so that $\mathbf{x}^{*}$ is feasible to it. If $\mathbf{x}^{*}$ does not solve $(7)$, then there exists $\tilde{\mathbf{x}} \in X$ satisfying $f(\tilde{\mathbf{x}}) \geq_{C} f\left(\mathbf{x}^{*}\right)$ such that $\mathbf{d}^{\mathrm{T}} f(\tilde{\mathbf{x}})>\mathbf{d}^{\mathrm{T}} f\left(\mathbf{x}^{*}\right)$. But then $f(\tilde{\mathbf{x}})-f\left(\mathbf{x}^{*}\right) \neq \mathbf{0}$ and so $f(\tilde{\mathbf{x}})>_{C} f\left(\mathbf{x}^{*}\right)$ in contradiction to the assumption that $\mathbf{x}^{*}$ solves problem (2). It follows that if $\mathbf{x}^{*}$ solves problem (2), then $\mathbf{x}^{*}$ solves problem (6) for $\varepsilon=f\left(\mathbf{x}^{*}\right)$.

To prove the sufficiency, let $\varepsilon \in R^{n}$ and assume that $\mathbf{x}^{*}$ solves problem (7). If $\mathbf{x}^{*}$ is not an efficient point for problem (2), then there exists $\hat{\mathbf{x}} \in X$ such that $f(\hat{\mathbf{x}})>_{c} f\left(\mathbf{x}^{*}\right)$. But since $\mathbf{x}^{*}$ is feasible to problem $(7)$ and satisfies $f\left(\mathbf{x}^{*}\right) \geq_{C} \varepsilon$, then $f(\hat{\mathbf{x}}) \geq_{C} \varepsilon$ and $\hat{\mathbf{x}}$ is also feasible to problem (7). Moreover, $f(\hat{\mathbf{x}})-f\left(\mathbf{x}^{*}\right) \in C \backslash\{\mathbf{0}\}$, so $\quad \mathbf{d}^{\mathrm{T}} f(\hat{\mathbf{x}})>\mathbf{d}^{\mathrm{T}} f\left(\mathbf{x}^{*}\right)$ in contradiction to the assumption that $\mathbf{x}^{*}$ solves (7). It follows that if $\mathbf{x}^{*}$ solves problem (7) for any $\varepsilon \in R^{n}$, then $\mathbf{x}^{*}$ solves problem (2). Choose any such $\varepsilon \in R^{n}$ to complete the proof.

The following corollary follows immediately from the above proof and states Theorem 10 as it is used.

Corollary 11. Let $C$ be a closed cone in $R^{n}$ and let $\mathbf{d} \in C^{+}$be given. If $\mathbf{x}^{*}$ solves problem (7) for any $\varepsilon \in R^{n}$, then $\mathbf{x}^{*}$ is an efficient point for problem (2). Moreover, for this given $\mathbf{d} \in C^{+}$, if $\mathbf{x}^{*}$ is an efficient point for (2), then $\mathbf{x}^{*}$ can be obtained from (7) for some $\varepsilon \in R^{n}$.

Note that an efficient point obtained via Theorem 10 or Corollary 11 need not be proper, in contrast to one obtained via Result 7, Corollary 8, and Corollary 9. This fact will be demonstrated in Example 16 of the next section. Thus for any fixed $\mathbf{d} \in C^{+}$, the vector parameter $\varepsilon$ of Corollary 11 can be varied to obtain at least theoretically the entire efficient frontier of problem (2). In addition, the parameters $\mathbf{d}$ and $\varepsilon$ together can further specify the decision maker's criterion than can the cone $C$ alone. For example, different $\mathbf{d} \in C^{+}$weight the importance of the objective functions $f_{1}(\mathbf{x}), \ldots, f_{n}(\mathbf{x})$ differently with respect to each other. In addition, the constraint $f(\mathbf{x}) \geq_{C} \varepsilon$ in problem (7) may reduce the number of choices. This constraint can be interpreted as the decision maker seeking a decision $\mathbf{x}^{*}$ for which the vector objective function $f\left(\mathbf{x}^{*}\right)$ achieves at least the vector aspiration level $\varepsilon$ with regard to $\geq_{C}$. On the other hand, if any $\varepsilon_{i}$ is sufficiently large in problem (7), no solution may exist, in which case the decision maker must reduce this aspiration level. Moreover, if all $\varepsilon_{i}$ are sufficiently small, the constraint $f(\mathbf{x}) \geq_{C} \varepsilon$ would not actually restrict a solution to (7).

Corollary 11 has obvious analogs to Corollaries 8 and 9 .

Corollary 12. Let $C$ be a polyhedral cone with basic generators $\mathbf{b}_{1}, \ldots, \mathbf{b}_{r}$. With $\mathbf{d} \in C^{+}$as specified in Corollary 8 under the conditions there for $\mathbf{b}_{1}, \ldots, \mathbf{b}_{r}$, for any $\varepsilon \in R^{n}$ if $\mathbf{x}^{*}$ solves problem $(7)$, then $\mathbf{x}^{*}$ is an efficient point for problem $(2)$.

Corollary 13. Let the $t \times n$ generator matrix $\mathbf{A}$ for the polyhedral cone $C$ have row vectors $\mathbf{a}_{1}, \ldots, \mathbf{a}_{t}$. For any $\mathbf{d}=\left(d_{1}, \ldots, d_{t}\right) \in R^{t}$ with $d_{i}>0, i=1, \ldots, t$, if $\mathbf{x}^{*}$ solves the scalar maximization problem

$$
\begin{gathered}
\operatorname{maximize} \sum_{i=1}^{t} d_{i} \mathbf{a}_{i} f(\mathbf{x}) \\
\text { subject to } \\
\left.\mathbf{a}_{i} f(\mathbf{x})\right) \geq \boldsymbol{\varepsilon}_{i}, i=1, \ldots, t \\
\mathbf{x} \in X
\end{gathered}
$$

for any $\varepsilon_{i}, i=1, \ldots, n$, then $\mathbf{x} *$ is an efficient point for problem (2).

A related result for basic generators is not a consequence of Corollary 11. Theorem 14 holds for the Pareto cone, among others.

Theorem 14. Let $C$ be a polyhedral cone in $R^{n}$ with basic generator $\mathbf{b}_{1}, \ldots, \mathbf{b}_{r}$ satisfying $\mathbf{b}_{i}{ }^{\mathrm{T}} \mathbf{b}_{j} \geq 0, i, j=1, \ldots, r$. For any $\varepsilon=\left(\varepsilon_{1}, \ldots, \varepsilon_{r}\right) \in R^{r}$ if $\mathbf{x}^{*}$ solves 


$$
\begin{gathered}
\operatorname{maximize} \sum_{i=1}^{r} \mathbf{b}_{i}{ }^{\mathrm{T}} f(\mathbf{x}) \\
\text { subject to } \\
\mathbf{b}_{i}{ }^{\mathrm{T}} f(\mathbf{x}) \geq \boldsymbol{\varepsilon}_{i}, i=1, \ldots, r \\
\mathbf{x} \in X
\end{gathered}
$$

then $\mathbf{x}^{*}$ is an efficient point for problem (2).

Proof. To prove the contrapositive, assume that $\mathrm{x}^{*} \in X$ is not an efficient point for problem (2). Then there exists $\hat{\mathbf{x}} \in X \quad$ such that $f(\hat{\mathbf{x}})-f\left(\mathbf{x}^{*}\right) \in C \backslash\{\mathbf{0}\}$. Hence $f(\hat{\mathbf{x}})-f\left(\mathbf{x}^{*}\right)=\sum_{j=1}^{r} \alpha_{j} \mathbf{b}_{j} \quad$ with $\alpha_{j} \geq 0, j=1, \ldots, r, \quad$ and $\quad$ some $\quad \alpha_{k}>0$. By assumption, $\quad \mathbf{b}_{i}{ }^{\mathrm{T}} \mathbf{b}_{j} \geq 0, i, j=1, \ldots, r, \quad$ from $\quad$ which $\mathbf{b}_{i}{ }^{\mathrm{T}}\left[f(\hat{\mathbf{x}})-f\left(\mathbf{x}^{*}\right)\right]=\sum_{j=1}^{r} \alpha_{j} \mathbf{b}_{i}{ }^{\mathrm{T}} \mathbf{b}_{j} \geq 0, i=1, \ldots, r$, and $\mathbf{b}_{k}{ }^{\mathrm{T}}\left[f(\hat{\mathbf{x}})-f\left(\mathbf{x}^{*}\right)\right]==\sum_{j=1}^{r} \alpha_{j} \mathbf{b}_{k}{ }^{\mathrm{T}} \mathbf{b}_{j}>0$. Thus

$$
\sum_{i=1}^{r} \mathbf{b}_{i}^{\mathrm{T}}\left[f(\hat{\mathbf{x}})-f\left(\mathbf{x}^{*}\right)\right]=\sum_{i=1}^{r} \mathbf{b}_{i}^{\mathrm{T}} f(\hat{\mathbf{x}})-\sum_{i=1}^{r} \mathbf{b}_{i}{ }^{\mathrm{T}} f\left(\mathbf{x}^{*}\right)>0
$$

Next set $\varepsilon_{i}=\mathbf{b}_{i}{ }^{\mathrm{T}} f\left(\mathbf{x}^{*}\right), i=1, \ldots, r$, in problem (9) so that from equation (10), $\hat{\mathbf{x}} \in X$ is feasible to (9). It now follows from equation (10) that $\mathbf{x}^{*} \in X$ does not solve problem (9). Thus if $\mathbf{x}^{*} \in X$ is not an efficient point for $(2)$, then there exist $\varepsilon_{i}, i=1, \ldots, r$, for which $\mathbf{x}^{*} \in X$ is not a solution to problem (9); and the proof is complete.

\section{Obtaining Vectors $\mathrm{d} \in C^{+}$}

In the previous section, for the scalarizations of Result 7, Corollary 8, and Theorem 10, as well as those of Corollary 11, and Corollary 12, a vector $\mathbf{d} \in C^{+}$is needed to compute a solution to problem (2) for a polyhedral cone. Corollaries 9 and 13 similarly require a vector $\mathbf{d} \in P^{+}$, but the condition there reduces to $\mathbf{d}>\mathbf{0}$, which presents no difficulty. Throughout this section, $C$ will be a polyhedral cone in $R^{n}$ described by either basic generators $\mathbf{b}_{1}, \ldots, \mathbf{b}_{r} \in R^{n}$ or an $t \times n$ generator matrix $\mathbf{A}$ with kernel $\{\mathbf{0}\}$.

We first discuss how to determine $C^{+}$when $\mathbf{A}$ is given. Recall that $C=\left\{\mathbf{y} \in R^{n}: \mathbf{A y} \geq \mathbf{0}\right\}$. It follows that the transposes of the nonzero row vectors $\mathbf{a}_{1}, \ldots, \mathbf{a}_{t} \in R^{n}$ of $\mathbf{A}$ are in the dual cone of $C$ by definition, as is any conical combination $\mathbf{d}=\sum_{j=1}^{t} \alpha_{j} \mathbf{a}_{j}^{\mathrm{T}}, \alpha_{j} \geq 0, j=1, \ldots, t$. If $\alpha_{j}>0, j=1, \ldots, t$, then $\mathbf{d} \in C^{+}$; otherwise the kernel of $\mathbf{A}$ is not $\{\mathbf{0}\}$. Hence, given the matrix $\mathbf{A}$, multiple vectors $\mathbf{d} \in C^{+}$ can be computed from $\mathbf{d}=\sum_{j=1}^{t} \alpha_{j} \mathbf{a}_{j}^{\mathrm{T}}, \alpha_{j} \geq 0, j=1, \ldots, t$. using different sets of positive $\alpha_{j}$. However, not all $\mathbf{d} \in C^{+}$have this form. As a counterexample in $R^{2}$, consider the generator matrix $\mathbf{A}=\left(\begin{array}{cc}1 & -1 \\ 0 & -1\end{array}\right)$ with kernel $\{\mathbf{0}\}$. For this example, obvious basic generators are the column vectors $\mathbf{b}_{1}=(1,1)$ and $\mathbf{b}_{2}=(0,-1)$. Then the column vector $\mathbf{d}=(2,-1)$ satisfies $\mathbf{d}^{\mathrm{T}} \mathbf{b}_{i}>0, i=1,2$, and is thus in $C^{+}$from Result 6. However, there do not exist $\alpha_{1}, \alpha_{2}>0$ such that $\left(\begin{array}{l}2 \\ 1\end{array}\right)=\alpha_{1}\left(\begin{array}{c}1 \\ -1\end{array}\right)+\alpha_{2}\left(\begin{array}{c}0 \\ -1\end{array}\right)$.

We next consider the case where $C$ is given in terms of basic generators $\mathbf{b}_{1}, \ldots, \mathbf{b}_{r} \in R^{n}$. For such a cone, we present an approach resembling the phase-one method of linear programming [22] to obtain vectors $\mathbf{d} \in C^{+}$to use in scalarizations (5) and (7), and hence their corollaries. A preliminary result is needed.

Result 15. If $\mathbf{d} \in R^{n}$ satisfies

$$
\mathbf{d}^{\mathrm{T}} \mathbf{b}_{i} \geq 1, i=1, \ldots, r
$$


then $\mathbf{d} \in C^{+}$. Moreover, there exists a $\mathbf{d} \in C^{+}$satisfying (11).

Proof. Suppose $\mathbf{d} \in R^{n}$ satisfies (11). Then immediately $\mathbf{d}^{\mathrm{T}} \mathbf{b}_{i}>0, i=1, \ldots, r$, and so $\mathbf{d} \in C^{+}$from Result 6. To show there exists $\mathbf{d}$ satisfying (11), note that there exists a $\hat{\mathbf{d}} \in C^{+}$from Result 6 satisfying $\quad \hat{\mathbf{d}}^{\mathrm{T}} \mathbf{b}_{i}>0, i=1, \ldots, r . \quad$ Let $\theta=\min _{i=1, \ldots, r} \hat{\mathbf{d}}^{\mathrm{T}} \mathbf{b}_{i}>0 . \quad$ Then $\quad \hat{\mathbf{d}}^{\mathrm{T}} \mathbf{b}_{i} \geq \theta, \mathrm{i}=1, \ldots, \mathrm{r}, \quad$ from $\quad$ which $\frac{\hat{\mathbf{d}}^{\mathrm{T}}}{\theta} \mathbf{b}_{i} \geq 1>0, \mathrm{i}=1, \ldots, \mathrm{r}$. Thus $\mathbf{d}=\frac{\hat{\mathbf{d}}}{\theta} \in C^{+}$is the required vector.

To formulate a linear programming problem for obtaining $\mathbf{d} \in C^{+}$, consider the $n \times r$ matrix $\mathbf{B}=\left[\mathbf{b}_{1}, \ldots, \mathbf{b}_{r}\right]$ with columns vectors $\mathbf{b}_{1}, \ldots, \mathbf{b}_{r}$. Let $\mathbf{1}$ denote the $r \times 1$ column vector $(1, \ldots, 1)$ and $\mathbf{S}$ be an $r \times 1$ column vector $\left(s_{1}, \ldots, s_{r}\right)$ of surplus variables. Then inequality (11) can be expressed in terms of $\mathbf{d}$ and $\mathbf{s}$ as

$$
\begin{gathered}
\mathrm{B}^{\mathrm{T}} \mathrm{d}-\mathrm{S}=\mathbf{1} \\
\mathrm{s} \geq \mathbf{0}
\end{gathered}
$$

where the inequality $\mathbf{s} \geq \mathbf{0}$ is interpreted componentwise. From Result 15, system (12) has a solution; and for any such solution $(\mathbf{d}, \mathbf{s})$, then $\mathbf{d} \in C^{+}$. To obtain this $\mathbf{d}$ numerically, define an $r \times 1$ column vector $\mathbf{v}=\left(v_{1}, \ldots, v_{r}\right)$ of artificial variables, and write the linear programming problem

$$
\begin{gathered}
\underset{\mathbf{d}, \mathbf{s}, \mathbf{v}}{\operatorname{minimize}} z=\mathbf{1}^{\mathrm{T}} \mathbf{v} \\
\text { subject to } \\
\mathbf{B}^{\mathrm{T}} \mathbf{d}-\mathbf{s}+\mathbf{v}=\mathbf{1} \\
\mathbf{s}, \mathbf{v} \geq \mathbf{0}
\end{gathered}
$$

From the previous discussion, if there exists a $\mathbf{d}$, together with $\mathbf{v}=\mathbf{0}$, and $\mathbf{s} \geq \mathbf{0}$ such that $(\mathbf{d}, \mathbf{s}, \mathbf{v})$ solves linear programming problem (13), then $\mathbf{d} \in C^{+}$. Moreover, a solution to (13) always exists since there exists $\mathbf{d} \in C^{+}$from Result 5. For any fixed $\hat{\mathbf{d}} \in C^{+}$, if $\mathbf{x}^{*}$ solves scalarization (5) or (7) with objective function $\hat{\mathbf{d}}^{\mathrm{T}} f(\mathbf{x})$, then $\mathbf{x}^{*}$ obviously solves either scalarization for $\frac{\hat{\mathbf{d}}}{\theta} \in C^{+}, \forall \theta>0$. Moreover, for $0<\theta=\min _{i=1, \ldots, r_{i}} \hat{\mathbf{d}}^{\mathrm{T}} \mathbf{b}_{i}$, there exist $\mathbf{s} \geq \mathbf{0}$ and $\mathbf{d}=\frac{\hat{\mathbf{d}}}{\theta} \operatorname{such}$ that $(\mathbf{d}, \mathbf{s}, \mathbf{0})$ solves problem (13). The preceding reasoning can be summarized as follows.

Theorem 16. If $\mathbf{d}$ is part of a solution $(\mathbf{d}, \mathbf{s}, \mathbf{0})$ to $(13)$, then $\mathbf{d} \in C^{+}$. Moreover, $\mathbf{x}^{*}$ is an efficient point for problem (2) obtained from scalarization (5) or (7) for some $\hat{\mathbf{d}} \in C^{+}$if and only if $\mathbf{x}^{*}$ can be obtained for (2) from (5) or (7) using some $\mathbf{d}$ in a solution (d,s, 0) to (13).

\section{Examples}

Some examples are next presented to illustrate the results of Sections 4 and 5 for polyhedral cones. These examples indicate the application and usefulness of the results. Without a scalarization, any maximization with respect to a non-Pareto cone could be extremely difficult to solve analytically. When the cone is polyhedral, the process is somewhat simpler, though not trivial as shown by the examples. Theorem 14 is particularly useful for many polyhedral cones in practice. It does not require any knowledge of the set $C^{+}$, which can be difficult to obtain.

Example 17. Consider the cone-ordered maximization problem

$$
\begin{gathered}
\text { Cmax } f(\mathbf{x})=\left(3 x_{1}+x_{2}, x_{1}+2 x_{2}\right) \\
\text { subject to } \\
2 x_{1}+3 x_{2} \leq 6 \\
x_{1}, x_{2} \geq 0
\end{gathered}
$$


where $C$ is generated by $\mathbf{b}_{1}=(1,1)$ and $\mathbf{b}_{2}=(-1,1)$. We solve problem (14) by scalarizations (5), (7) and (9). Set $X=\left\{\left(x_{1}, x_{2}\right): 2 x_{1}+3 x_{2} \leq 6 ; x_{1}, x_{2} \geq 0\right\}$. To use (5), we must find some $\mathbf{d}=\left(d_{1}, d_{2}\right) \in C^{+}$. Since Result 6 requires that $\mathbf{d}^{\mathrm{T}} \mathbf{b}_{i}>0, i=1, \ldots, r$, it follows here that $\mathbf{d}^{\mathrm{T}} \mathbf{b}_{1}=d_{1}+d_{2}>0$ and $\mathbf{d}^{\mathrm{T}} \mathbf{b}_{2}=-d_{1}+d_{2}>0$. Hence $C^{+}=\left\{\left(d_{1}, d_{2}\right):-d_{2}<d_{1}<d_{2}\right\}$ for problem (14). A vector $\mathbf{d} \in C^{+}$can also be obtained by solving linear programing problem (13), which becomes here

$$
\begin{gathered}
\underset{d_{1}, d_{2}, s_{1}, s_{2}, v_{1}, v_{2}}{\operatorname{minimize}} z=v_{1}+v_{2} \\
\text { subject to } \\
d_{1}+d_{2}-s_{1}+v_{1}=1 \\
-d_{1}+d_{2}-s_{2}+v_{2}=1 \\
s_{1}, s_{2}, v_{1}, v_{2} \geq 0
\end{gathered}
$$

Then $\mathbf{d} \in C^{+}$is the $\mathbf{d}^{*}$ in a solution $\left(\mathbf{d}^{*}, \mathbf{s}^{*}, \mathbf{0}\right)$. Solving linear programming problem (15) with the IBM CPLEX 12.5 (or any linear programming solver) yields $d_{1}=0, d_{2}=1$ as one solution. In general, multiple extreme point solutions $(\mathbf{d}, \mathbf{s}, \mathbf{0})$ of (13) can be obtained in CPLEX by using the alternate optima option. Then for any two optimal extreme points $\left(\mathbf{d}_{1}, \mathbf{s}_{1}, \mathbf{0}\right),\left(\mathbf{d}_{2}, \mathbf{s}_{2}, \mathbf{0}\right)$ and all $0<\lambda<1$, the vector $\lambda \mathbf{d}_{1}+(1-\lambda) \mathbf{d}_{2} \in C^{+}$. Using $\mathbf{d}=(0,1)$ computed above and solving (14) by scalarization (5) gives

$$
\begin{gathered}
\operatorname{maximize} \mathrm{z}=x_{1}+2 x_{2} \\
\text { subject } \text { to } \\
2 x_{1}+3 x_{2} \leq 6 \\
x_{1}, x_{2} \geq 0
\end{gathered}
$$

whose solution $\left(x_{1}, x_{2}\right)=(0,2)$ is a proper efficient point for (14) from Result 7 .

For any $\mathbf{d} \in C^{+}$, scalarization (5) for problem (14) has a single constraint, other than the nonnegativity ones, and thus from the theory of linear programming [22] has a single basic variable. Thus in (16) $x_{1}$ and $x_{2}$ cannot both be positive unless there are alternate nonbasic solutions, which there are not. Hence, the only candidates $\left(x_{1}, x_{2}\right)$ for being solutions and proper efficient points for (12) are $(0,2),(3,0)$, or $(0,0)$. Of course, $(0,2)$ was obtained above, and $(3,0)$ could be similarly obtained for $d_{1}=1, d_{2}=2$. On the other hand, it can be easily shown that $(0,0)$ cannot solve (5) for any $\mathbf{d}=\left(d_{1}, d_{2}\right) \in C^{+}$. Now note that $X$ is a convex set in problem (12). Moreover, $f$ is linear in both its objective functions and is therefore C-concave on a convex $X$. From Result $7, \mathbf{x}^{*}$ is a proper efficient point for problem (14) if and only if $\mathbf{x}^{*}$ solves problem (5) for some $\mathbf{d} \in C^{+}$. Thus any efficient point for $(14)$ besides $(0,2)$ or $(3,0)$ must be improper. We now find one.

Since $\mathbf{b}_{1}=(1,1)$ and $\mathbf{b}_{2}=(-1,1)$ satisfy the hypotheses of Theorem 14, we can solve problem (12) by scalarization (9), which yields

$$
\begin{gathered}
\text { maximize } \mathrm{z}=2 x_{1}+4 x_{2} \\
\text { subject to } \\
4 x_{1}+3 x_{2} \geq \varepsilon_{1} \\
-2 x_{1}+x_{2} \geq \varepsilon_{2} \\
2 x_{1}+3 x_{2} \leq 6 \\
x_{1}, x_{2} \geq 0
\end{gathered}
$$

The solution to the linear programming problem (17) is readily obtained to be $\left(x_{1}, x_{2}\right)=(0,2)$ for $\left(\varepsilon_{1}, \varepsilon_{2}\right)=(1,1)$ and $\left(x_{1}, x_{2}\right)=(3,0)$ for $\left(\varepsilon_{1}, \varepsilon_{2}\right)=(10,-10)$. However, for $\left(\varepsilon_{1}, \varepsilon_{2}\right)=(9,-8)$ the solution is $\left(x_{1}, x_{2}\right)=(3 / 2,1)$, which must be an improper efficient point as noted above since it could not be obtained by scalarization (5). 
Finally, we solve (14) by scalarization (7). Again let $\mathbf{d}=(0,1) \in C^{+}$to yield

$$
\begin{gathered}
\text { maximize } \mathrm{z}=x_{1}+2 x_{2} \\
\text { subject to } \\
\left(3 x_{1}+x_{2}, x_{1}+2 x_{2}\right) \geq_{C}\left(\varepsilon_{1}, \varepsilon_{2}\right) \\
2 x_{1}+3 x_{2} \leq 6 \\
x_{1}, x_{2} \geq 0
\end{gathered}
$$

The constraint $\left(3 x_{1}+x_{2}, x_{1}+2 x_{2}\right) \geq_{C}\left(\varepsilon_{1}, \varepsilon_{2}\right)$ cannot interpreted componentwise because of the cone order $\geq_{C}$. To handle this constraint, write $\left(3 x_{1}+x_{2}-\varepsilon_{1}, x_{1}+2 x_{2}-\varepsilon_{2}\right) \in C$, which has basic generators $\mathbf{b}_{1}=(1,1)$ and $\mathbf{b}_{2}=(-1,1)$. From the definition of the basic generators, it follows that for any $\left(\varepsilon_{1}, \varepsilon_{2}\right)$ and $\left(x_{1}, x_{2}\right)$ the point $\left(3 x_{1}+x_{2}-\varepsilon_{1}, x_{1}+2 x_{2}-\varepsilon_{2}\right)$ in $C$ can be written as a conical combination of the generators $(1,1)$ and $(-1,1)$, i.e.,

$$
\left(3 x_{1}+x_{2}-\varepsilon_{1}, x_{1}+2 x_{2}-\varepsilon_{2}\right)=\alpha_{1}(1,1)+\alpha_{2}(-1,1)
$$

for some $\alpha_{1}, \alpha_{2} \geq 0$. Next rewrite equation (19) componentwise as

$$
\begin{aligned}
& 3 x_{1}+x_{2}-\varepsilon_{1}=\alpha_{1}-\alpha_{2} \\
& x_{1}+2 x_{2}-\varepsilon_{2}=\alpha_{1}+\alpha_{2}
\end{aligned}
$$

Replacing the first equation in (20) by the sum of both equations gives

$$
\begin{aligned}
& 4 x_{1}+3 x_{2}-\varepsilon_{1}-\varepsilon_{2}=2 \alpha_{1} \geq 0 \\
& x_{1}+2 x_{2}-\varepsilon_{2}=\alpha_{1}+\alpha_{2} \geq 0
\end{aligned}
$$

Fix $\left(\varepsilon_{1}, \varepsilon_{2}\right)$. Then $(21)$ is equivalent to restricting $\left(x_{1}, x_{2}\right)$ to satisfying $(19)$; i.e.,

$$
\begin{aligned}
4 x_{1}+3 x_{2} & \geq \varepsilon_{1}+\varepsilon_{2} \\
x_{1}+2 x_{2} & \geq \varepsilon_{2}
\end{aligned}
$$

In other words, for fixed $\left(\varepsilon_{1}, \varepsilon_{2}\right)$ the point $\left(x_{1}, x_{2}\right)$ satisfies (22) if and only if there exist $\alpha_{1}, \alpha_{2} \geq 0$ satisfying (19). Thus replacing $\left(3 x_{1}+x_{2}, x_{1}+2 x_{2}\right) \geq_{C}\left(\varepsilon_{1}, \varepsilon_{2}\right)$ in (18) by (22) and solving scalarization (7) with $\mathbf{d}=(0,1)$ yields

$$
\begin{gathered}
\text { maximize } \mathrm{z}=x_{1}+2 x_{2} \\
\text { subject to } \\
4 x_{1}+3 x_{2} \geq \varepsilon_{1}+\varepsilon_{2} \\
x_{1}+2 x_{2} \geq \varepsilon_{2} \\
2 x_{1}+3 x_{2} \leq 6 \\
x_{1}, x_{2} \geq 0
\end{gathered}
$$

Setting $\left(\varepsilon_{1}, \varepsilon_{2}\right)=(1,1)$ and solving linear programming problem $(23)$ gives the solution $\left(x_{1}, x_{2}\right)=(0,2)$, while $\left(\varepsilon_{1}, \varepsilon_{2}\right)=(9,3)$ yields $\left(x_{1}, x_{2}\right)=(3,0)$. On the other hand, for $\left(\varepsilon_{1}, \varepsilon_{2}\right)=(4,4)$ there is no feasible solution to problem $(23)$

Example 18. Now consider the cone-ordered maximization problem

$$
\begin{gathered}
C \max f(\mathbf{x})=\left(-x_{1} x_{2}+4 x_{3}, 2 x_{1}+5 x_{2} x_{3}, 3 x_{2}+4 x_{1} x_{3}\right) \\
\text { subject to } \\
x_{1}^{2}+x_{2}^{2}+x_{3}^{2} \leq 27 \\
x_{1}+2 x_{2}+3 x_{3}=18 \\
x_{1}, x_{2}, x_{3} \geq 0
\end{gathered}
$$

where $C$ is generated by $\mathbf{b}_{1}=(1,0,0), \mathbf{b}_{2}=(1,0,1), \mathbf{b}_{3}=(0,1,1), \mathbf{b}_{4}=(0,1,0)$ in $R^{3}$ for $r=4>n=3$. We set $X=\left\{\left(x_{1}, x_{2}, x_{3}\right): x_{1}{ }^{2}+x_{2}{ }^{2}+x_{3}{ }^{2} \leq 27 ; x_{1}+2 x_{2}+3 x_{3}=18 ; x_{1}, x_{2}, x_{3} \geq 0\right\}$ and use Theorem 14 to write scalarization (9) for problem (24) as 


$$
\begin{gathered}
\operatorname{maximize} \mathrm{z}=4 x_{1}-2 x_{1} x_{2}+8 x_{1} x_{3}+6 x_{2}+8 x_{3}+10 x_{1} x_{3} \\
\text { subject to } \\
-x_{1} x_{2}+4 x_{3} \geq \varepsilon_{1} \\
-x_{1} x_{2}+3 x_{2}+4 x_{3}+4 x_{1} x_{3} \geq \varepsilon_{2} \\
2 x_{1}+3 x_{2}+5 x_{1} x_{2}+4 x_{1} x_{3} \geq \varepsilon_{3} \\
x_{1}{ }^{2}+x_{2}{ }^{2}+x_{3}{ }^{2} \leq 27 \\
x_{1}+2 x_{2}+3 x_{3}=18 \\
x_{1}, x_{2}, x_{3} \geq 0
\end{gathered}
$$

Let $\left(\varepsilon_{1}, \varepsilon_{2}, \varepsilon_{2}\right)=(3,48,96)$. For these values, problem (25) has a continuous (nonconcave) objective function on a closed, bounded, nonempty feasible region. It thus has a solution. The problem was solved with Mathematica using both the simulated annealing and Nelder-Mead methods, as well as with Excel's generalized reduced gradient solver. In all cases, to three decimal places $\left(x_{1}, x_{2}, x_{3}\right)=(3.100,2.756,3.129)$ was found to be the associated efficient point for problem (22). Other efficient points can be found by varying $\left(\varepsilon_{1}, \varepsilon_{2}, \varepsilon_{2}\right)$.

Example 19. Consider again the cone-ordered maximization problem (14) of Example 17. Now use the generator matrix $\mathbf{A}=\left(\begin{array}{cc}1 & 1 \\ -1 & 1\end{array}\right)$ of the polyhedral cone $C$. Then scalarization (6) becomes

$$
\begin{gathered}
\text { maximize } \mathrm{z}=d_{1}\left(4 x_{1}+3 x_{2}\right)+d_{2}\left(-2 x_{1}+x_{2}\right) \\
\text { subject to } \\
2 x_{1}+3 x_{2} \leq 6 \\
x_{1}, x_{2} \geq 0
\end{gathered}
$$

We use $\mathbf{d}=(0,2) \in C^{+}$from $\mathbf{d}=\mathbf{a}_{1}^{\mathrm{T}}+\mathbf{a}_{2}^{\mathrm{T}}$ as discussed in Section 5. Then (26) becomes

$$
\begin{gathered}
\text { maximize } \mathrm{z}=2 x_{1}+4 x_{2} \\
\text { subject } \text { to } \\
2 x_{1}+3 x_{2} \leq 6 \\
x_{1}, x_{2} \geq 0
\end{gathered}
$$

Solving (27) by linear programming gives a solution $(0,2)$. The solution $(3,0)$ could be obtained with another $\mathbf{d}$

\section{Conclusions}

It is likely that most practical applications of cone-ordered maximization will involve polyhedral cones to model multiobjective decision criteria. These cones can be defined either by generator sets or by generator matrices. The former appears more intuitive for defining an appropriate cone for a model. A practitioner need only choose various generator sets by trial and error until an appropriate cone is determined. Regardless, all scalarizations presented here for polyhedral cones, except (9) of Theorem 14, require a dual vector $\mathbf{d} \in C^{+}$for the scalarized objective function. For this reason, approaches for obtaining these vectors were presented for both generator sets and generator matrices. In particular, for $C$ described by a generator set, a linear programming approach was presented for obtaining dual vectors $\mathbf{d} \in C^{+}$such that all efficient points for (2) calculated using scalarization (5) and (7) can theoretically be computed. Examples were given to illustrate the theory and reasoning involved in solving problems.

\section{References}

1. L. Hurwicz, "Programming in linear spaces," Studies in linear and nonlinear programming, edited by K.J. Arrow,

L. Hurwicz, and H. Uzawa, Stanford University Press, pp. 38-102, 1958. 
2. L.W. Neustadt, "A general theory of extremals," Journal of computer and system sciences, vol. 3, pp. 57-92, 1969.

3. K. Ritter, "Optimization theory in linear spaces III: Mathematical programming in partially ordered Banach spaces," Mathematische annalen, vol. 184, pp. 133-154, 1970.

4. B.D. Craven, "Nonlinear programming in locally convex spaces," Journal of optimization theory and applications, vol. 10, pp. 197-210, 1972.

5. P.L. Yu, "Cone convexity, cone extreme points, and nondominated solutions in decision problems with multiobjectives," Journal of optimization theory and applications, vol. 14, pp. 319-377, 1974.

6. J. Borwein, "Proper efficient points for maximizations with respect to cones," SIAM journal on control and optimization, vol. 15, pp. 57-63, 1977.

7. N. Christopeit, "Necessary optimality conditions with applications to a variational problem," SIAM journal on control and optimization, vol. 15, pp. 683-698, 1977.

8. H.W. Corley, "On optimality conditions for maximizations with respect to cones," Journal of optimization theory and applications, vol. 46, pp. 67-78, 1985.

9. H.W. Corley, "Duality theory for maximizations with respect to cones," Journal of mathematical analysis and applications, vol. 84, pp. 560-568, 1981.

10. J. Jahn, "Scalarization in vector optimization," Mathematical programming, vol. 29, pp. 203-218, 1984.

11. R.M. Soland, "Multicriteria optimization: a general characterization of efficient solutions," Decision sciences, vol. 10, pp. 26-38, 1979.

12. P.L. Yu, Multiple-criteria decision making. Plenum Press, 1985.

13. Y. Sawaragi, H. Nakavama, and T. Tanino, Theory of multiobjective optimization. Academic Press, 1985.

14. Y. Collette and P. Siarry, Multiobjective optimization: principles and case studies. Springer, 2003.

15. M. Ehrgott, Multicriteria optimization. Springer, 2005.

16. J. Jahn, Vector optimization - theory, applications, and extensions. Springer, 2011.

17. A. Auslender and M. Teboulle, Asymptotic cones and functions in optimization and variational inequalities, Springer, 2015.

18. S.M. Grad, Vector optimization and monotone operators via convex duality: recent advances. Springer, 2015.

19. C. Gutierrez, L. Huerga, and V. Novo, "Nonlinear scalarization in multiobjective optimization with a polyhedral ordering cone," International transactions in operational research, doi:10.1111/itor.12398, 2017.

20. C. Davis, "Theory of positive dependence," American journal of mathematics, vol. 76, pp. 733-746, 1954.

21. J.Dattorro, Convex optimization and Euclidean distance geometry. Lulu.com, 2010.

22. A. Schrijver, Theory of linear and integer programming. John Wiley and Sons, 1986.

23. A.M. Geoffrion, "Proper efficiency and the theory of vector maximization," Journal of mathematical analysis and applications, vol. 22, pp. 618-630, 1968.

24. M.I. Henig, "Proper efficiency with respect to cones," Journal of optimization theory and applications, vol. 36, pp. 387-407, 1982 .

25. H.P. Benson, "Efficiency and proper efficiency in vector maximization with respect to cones," Journal of mathematical analysis and applications, vol. 93, pp. 273-289, 1983.

26. X. Zheng and X. Yang, "The structure of weak Pareto solution sets in piecewise linear multiobjective optimization in normed spaces," Science in China series A:mathematics, vol. 51, pp. 1243-1256, 2008.

27. R. Kasimbeyli, "A nonlinear cone separation theorem and scalarization in nonconvex vector optimization," SIAM Journal of optimization, vol. 20, pp. 1591-1619, 2010.

28. R Kasimbeyli, "A conic scalarization method in multi-objective optimization," Journal of global optimization, vol. 56, pp. 279-297, 2013.

29. K. Q. Zhao, Y.M. Xia, and Y.M. Yang, "Nonlinear scalarization characterization of E-efficiency in vector optimization," Taiwan journal of mathematics, vol. 19, pp. 455-466, 2015.

30. K.Q. Zhao, G.Y. Chen, and X.M. Yang, "Approximate proper efficiency in vector optimization," Optimization, vol. 64, pp. 1777-1793, 2015.

31. K.Q. Zhao and X.M. Yang, "E-Benson proper efficiency in vector optimization," Optimization, vol. 64, pp. 739$752,2015$.

32. C-R Chen, X. Zuo, F. Lu, and S-J Li, "Vector equilibrium problems under improvement sets and linear scalarization with stability applications," Optimization methods and software, vol. 31, pp. 1240-1257, 2016. 
33. H. Guo, and W.J. Zhang, "Conic scalarizations for approximate efficient solutions in nonconvex vector optimization problems," Journal of the operations research society of China, pp. 1-12, 2016.

34. K. Khaledian, E. Khorram, and M. Soleimani-damaneh, "Strongly proper efficient solutions: efficient solutions with bounded trade-offs," Journal of Optimization theory and applications, vol. 168, pp. 864-883, 2016.

35. S.Shahbeyk and M.Soleimani-damaneh, "Proper minimal points of nonconvex sets in Banach spaces in terms of the limiting normal cone," Optimization, vol. 66, pp. 473-489, 2017.

36. A. Engau, "Proper efficiency and tradeoffs in multiple criteria and stochastic optimization," Mathematics of operations research, vol. 42, pp. 119-134, 2017.

37. K.Q. Zhao, W.D.Rong, and X.M. Yang, "New nonlinear separation theorems and applications in vector optimization," Scientia sinica mathematica, vol. 47, pp. 533-544, 2017.

38. C. Gutierrez, B. Jimenez, and V. Novo, "Improvement sets and vector optimization," European journal of operations research, vol. 223, pp. 304-311, 2017.

39. E-D Rahmo and M. Studniarski, "A new global scalarization method for multiobjective optimization with an arbitrary ordering cone," Applied mathematics, vol. 8, pp. 154-163, 2017. 